\title{
Transient thromboembolic event in a child with severe hemophilia A due to venous obstruction
}

Athugalpura DMAA, Jayathilaka MM, Jayathilaka A, Gunathilaka PKG, Kankananarachchi I, Gamhewage N

\begin{abstract}
Venous thrombosis is a very rare occurrence in patients with hemophilia A. We present a one year old child with severe hemophilia $A$, who developed deep vein thrombosis in antecubital fossa following venous obstruction due to bleeding around the vein. We didn't start anticoagulant therapy to our patient because of the risk of further bleeding into subdural hemorrhage he had already developed. We could manage the thrombosis and save the limb by relieving the obstruction with replacement of factor VIII.
\end{abstract}

\section{Introduction}

Hemophilia $A$ is a hereditary hemorrhagic disease characterized by deficiency of coagulation Factor VIII. Venous thrombosis in patients with hemophilia $A$ has been rarely encountered in medical literature $[1,2$, 4-7] and it is an important cause of hospital acquired morbidity and mortality [3]. In this case report we present the onset of deep vein thrombosis in a one year old child with hemophilia A.

\section{Case report}

One year old boy, who had the diagnosis of severe hemophilia A (Factor level $0.286 \%$ ), and a past history of spontaneous intra cranial hemorrhage at 8 months of age, has presented with 2 days history of fever, reduced activity and drowsiness. CT scan revealed acute sub dural hemorrhage in falx \& in tentorium and chronic hemorrhage in left sided parieto-occipital region. He was on prophylactic factor viii weekly prior to current admission because of the previous spontaneous intra cranial hemorrhage. According to guidelines we started giving $100 \%$ factor viii correction twice daily to overcome further bleeding into sub dural space.

In spite of these measures, APTT remained elevated between $80 \mathrm{~s}$ and $90 \mathrm{~s}$ and the child had been less active and irritable. On day 9 of the ward stay, child has developed gross left upper limb swelling and we suspected him as having a local bleed followed by venous obstruction. Doppler ultrasonography showed 'Deep vein thrombosis in antecubital fossa without evidence

Lady Ridgeway Children Hospital of arterial compromise'. The vein at antecubital fossa in which the thrombosis developed was almost completely occluded due to hematoma around it. Activated partial thromboplastin time, bleeding time and other investigations are given in table 1

We decided to continue factor viii correction without anticoagulation because of the risk of further bleeding. Instead, we increased the dose of factor VIII. In addition, we gave a dose of prothrombin complex concentrate (PCC). At the same time blood was taken for 'Bethesda test' because we suspected inhibitors to factor VIII.

PT - prothrombin time, APTT - activated partial thromboplastin time, INR - international normalization ratio, WBC - white cell count, CRP - c reactive protein, AST - aspartate aminotransferase, ALT - alanine aminotransferace

Fresh frozen plasma infusion was given to overcome possible protein $\mathrm{C}$ or $\mathrm{S}$ deficiency. Vascular surgical team didn't want to operate and do the thrombectomy because of the risk of bleeding. We were able to manage limb edema conservatively while replacing factors. Limb was elevated and kept at higher level throughout the day.

Child was kept in the High dependency area of the ward, and closely monitored for the possible development of compartment syndrome. Daily coagulation test and monitoring of thrombocyte level were performed. With time, edema of the limb gradually settled, range of movements increased, and drowsiness improved. Followup Doppler ultrasonography showed 'normal L/upper limb vasculature with no thrombus, and normal blood flow observed in the affected limb. With improvement of the patient, factor viii dosing frequency was reduced and omitted. We decided to give weekly prophylactic 'Factor viii inhibitor bypassing agent' (prothrombin complex concentrate) to avoid spontaneous intra cranial bleeding.

\section{Discussion}

Venous thrombosis is an important cause of hospital acquired morbidity and mortality [3]. It is associated rarely with patients of hemophilia $A[1,2,4-7]$. Several risk factors have been described in medical literature. There were hardly any cases with normal coagulation test results at the time of thrombosis. The most important risk factor for patients with hemophiliaA was taking Factor VIII inhibitor bypassing activity (FEIBA) or recombinant 
activated factor VII (rFVIla) for inhibitors [3]. Other risk factors were congenital prothrombotic conditions, deficiency of protein C and Factor V Leiden [8], following total hip and knee arthroplasty [9], following insertion of central venous catheters [10]. Murat Bicer et al reported a spontaneous deep vein thrombosis originated from the right main and external iliac veins in a32 year old white male hemophilia A [11]. Van der Planken reported a deep venous thrombosis development within 18 days after recombinent activated factor VII (rFVIla) infusion in a 38 years old patient with hemophilia A [12]. Ettingshausen et al. described portal venous thrombosis in a patient with the diagnosis of hemophilia A and Factor V G1691A mutation [6].

Therearenoclearconsensusesregarding the management of thromboembolism in patients with hemophilia A because of the low number of cases in medical literature [11]. Dargaud et al. have used unfractioned heparin for a month subsequent to Factor VIII replacement [1]. Low molecular weight heparin was administered for 9 weeks by Kashyap et al [8]; Ettinsghausen et al had practiced different way of approach where he applied low molecular weight heparin together with Factor VIII replacement, after unfractioned heparin for 14 days.

Murat Bicer et al initiated oral anticoagulant drug after 48 hours of thrombosis because coagulation tests were normal at that time and Factor V Leiden was absent. Factor VIII levels were replaced during patient's follow-up. He suggested in his case report, to start oral anticoagulants with close monitoring for hemorrhage. In our case we were reluctant to start any anticoagulant, because APTT was never normal. It remained in very higher value. On the other hand patient had already developed sub dural hemorrhage which can aggravate with anticoagulation. Our aim was to cure the hematoma around the vessel relieving the obstruction. We expected spontaneous resolution of thrombosis when we remove the risk factor for thrombosis. Finally we were able to save the limb without any anticoagulant.

\section{References}

1. Dargaud Y, Cruchaudet BB, Lienhart A, Coppere B, Ninet J, Negrier $C$ : Spontaneous proximal deep vein thrombosis in a patient with severe haemophilia $\mathrm{A}$. Blood Coagul Fibrinolysis 2003, 14:407-409.

2. Girolami A, Scandellari R, Zanon E, Sartori R, Girolami $B$ : Noncatheter associated venous thrombosis in haemophilia A and B. A critical review of all reported cases. J Thromb Thromolysis 2006, 21:279-284.
3. Butcher JH, Pasi KJ: Fatal postoperative pulmonary embolism in mild haemophilia. Haemophilia 2006, 12:179-182.

4. Stewart AJ, Manson LM, Dennis R, Allan PL, Ludlam CA: Thrombosis in a duplicated superficial femoral vein in a patient with haemophilia A. Haemophilia 2000, 6:47-49.

5. Oclay L, Gurgey A, Topaloglu H, Atay S, Parlak H, Firat $\mathrm{M}$ : Cerebral infarction associated with factor $\mathrm{V}$ Leiden mutation in a boy with haemophilia A. Am J Hematol 1997, 58:189-190.

6. Ettingshausen $\mathrm{CE}$, Saguer IM, Kreuz W: Portal vein thrombosis in a patient with severe haemophilia $A$ and F V G1691A mutation during continuous infusion of F VIII after intramural jejunal bleeding-successful thrombolysis under heparin therapy. Eur J Pediatr 1999, 158:180-182.

7. Pruthi RK, Heit JA, Green MM, Emiliusen LM, Nichols WL, Wilke JL, Gastineau DA: Venous thromboembolism after hip fracture surgery in a patient with haemophilia B and factor V Arg $506 \mathrm{Gln}$ (factor V Leiden). Haemophilia 2006, 6:631-634.

8. Kashyap R, Sharma Lm, Gupta S, Saxena R, Srivastava $D N$ : Deep vein thrombosis in a patient with severe haemophilia A. Haemophilia 2006, 12:87-89.

9. Matthew I.Stein, MD; Justin park, MD; Stephen Raterman, MD, Prevention of VTE Following Total Hip and Knee Arthroplasty in Hemophilia Patients: cme Article, MAY 2011 |Volume 34 • Number 5: 389 $-391$

10. V.E. price, M. cargo, B. Connolly, P. charith,* A.daneman $m$ temple, A. M. stain, L sung Hal-tralbosi and V. S. Blanchet: A prospective, longitudinal study of central venous catheter related deep venous thrombosis in boys with hemophilia: Journal of Thrombosis and Haemostasis, 2: 737-742

11. Murat Bicer, Murat Yanar and Oktay Tuydes: Spontaneous deep vein thrombosis in hemophilia A: a case report: Cases Journal 2009, 2:6390:

12. Van der Planken MG, Schrovens $W$, Vertessen $F$, Michiels JJ, Berneman ZN: Distal deep venous thrombosis in a heamophilia A patient with inhibitor and severe infectious disease, 18 days after recombinant activated factor VII transfusion. Blood Coagul Fibrinolysis 2002, 13:367-370. 